\title{
Competitive technologies of third generation infrared photon detectors
}

\author{
A. ROGALSKI* \\ Institute of Applied Physics, Military University of Technology, 2 Kaliskiego Str., 00-908 Warsaw, Poland
}

Hitherto, two families of multielement infrared (IR) detectors are used for principal military and civilian infrared applications; one is used for scanning systems (first generation) and the other is used for staring systems (second generation). Third generation systems are being developed nowadays. In the common understanding, third generation IR systems provide enhanced capabilities like larger number of pixels, higher frame rates, better thermal resolution as well as multicolour functionality and other on-chip functions.

In the paper, issues associated with the development and exploitation of materials used in fabrication of third generation infrared photon detectors are discussed. In this class of detectors two main competitors, $\mathrm{HgCdTe}$ photodiodes and quantum well IR photoconductors (QWIPs) are considered. The performance figures of merit of state-of-the-art HgCdTe and QWIP focal plane arrays (FPAs) are similar because the main limitations come from the readout circuits. However, the metallurgical issues of the epitaxial layers such as uniformity and number of defected elements are the serious problems in the case of long wavelength infrared (LWIR) and very LWIR (VLWIR) HgCdTe FPAs. It is predicted that superlattice based InAs/GaInSb system grown on GaSb substrate seems to be an attractive to $\mathrm{HgCdTe}$ with good spatial uniformity and an ability to span cutoff wavelength from 3 to $25 \mu \mathrm{m}$.

Keywords: $\mathrm{HgCdTe}$ detectors, QWIPs, type II superlattices, third generation detectors.

\section{Introduction}

Multicolour capabilities are highly desirable for advance infrared (IR) systems [1-4]. Systems that gather data in separate IR spectral bands can discriminate both absolute temperature and unique signatures of objects in the scene. By providing this new dimension of contrast, multiband detection also enables advanced colour processing algorithms to further improve sensitivity above that of single-colour devices. This is extremely important for the process of identifying temperature difference between missile target, war heads, and decoys. Multispectral IR focal plane arrays (FPAs) can also play many important roles in Earth and planetary remote sensing, astronomy, etc. Currently, multispectral systems rely on cumbersome imaging techniques that either disperse the optical signal across multiple IR FPAs or use a filter wheel to spectrally discriminate the image focused on single FPA. These systems contain beamsplitters, lenses, and bandpass filters into the optical path to focus the images onto separate FPAs responding to different IR bands. Also complex alignment is required to map the multispectral image pixel for pixel. Consequently, these approaches are expensive in terms of size, complexity, and cooling requirements.

Both HgCdTe photodiodes [5-7] and quantum well infrared photodetectors (QWIPs) [7-10] offer the multicolour capability in the MWIR and LWIR range. The performance

*e-mail: rogan@wat.edu.pl figures of merit of state-of-the-art QWIP and $\mathrm{HgCdTe}$ FPAs are similar because the main limitations come from the readout circuits. A more detailed comparison of both technologies has been given by Tidrow et al. [7] and Rogalski [6,9].

Recently, type II InAs/GaInSb superlattices have emerged as third candidate for third generation infrared detectors [11-14]. Whether the superlattice IR photodetectors can outperform the "bulk" narrow gap HgCdTe detectors is one of the most important questions for the future of IR photodetectors.

\section{Requirements of third generation infrared systems}

Two families of multielement detectors can be considered for principal military and civilian IR applications; one used for scanning systems and the other used for staring systems. The scanning system, which does not include multiplexing functions in the focal plane, belongs to the first generation systems.

The second generation systems (full-framing systems) have typically three orders of magnitude more elements $\left(>10^{6}\right)$ on the focal plane than first generation systems and the detectors elements are configured in a two-dimensional (2-D) arrays. These staring arrays are scanned electronically by circuits integrated with the arrays. These devices are 2-D arrays of photodiodes connected with indium bumps to a readout integrated circuit (ROIC) chip as a hy- 
brid structure, often called a sensor chip assembly (SCA). Development of this technology began in the late 70's last century and took the next decade to reach volume production. In the early 1990's, fully 2-D arrays provided a means for staring sensor systems to begin production. Large IR detector arrays are now available that meet the demanding requirements of the astronomy and civil space applications. Astronomers in particular have eagerly waited for the day when electronic arrays could match the size of photographic film. Development of large format, high sensitivity, mosaic IR sensors for ground-based astronomy is the goal of many observatories around the world (large arrays dramatically multiply the data output of a telescope system). This is somewhat surprising given the comparative budgets of the defence market and the astronomical community.

For the last 25 years, array size has been increasing at an exponential rate, following a Moore's Law grow path (see Fig. 1), with the number of pixels doubling every 19 months. The graph shows the low of the number of pixels per SCA as a function of the year first used on astronomy for MWIR SCAs. Arrays are expected to exceed $4 \mathrm{~K} \times 4 \mathrm{~K}$ format - 16 million pixels - this year, about a year later than the Moore's Law prediction.

The trend of increasing pixel's number is likely to continue in the area of large format arrays. This increasing will be continued using close-butted mosaic of several SCAs as shown in Fig. 2. Raytheon manufactured a $4 \times 4$ mosaic of $2 \mathrm{~K} \times 2 \mathrm{~K} \mathrm{HgCdTe}$ SCAs and assisted in assembling into the final focal-plane configuration (see Fig. 2) to survey the entire sky in the Southern Hemisphere at four IR wavelengths. With 67 million pixels, this is currently the world's largest IR focal plane. Although there are currently limitations to reduce the size of the gaps between active detectors on adjacent SCAs, many of these can be overcome. It is predicted that focal plane of 100 megapixels and larger will be possible, constrained only by budgets, but not technology [15].

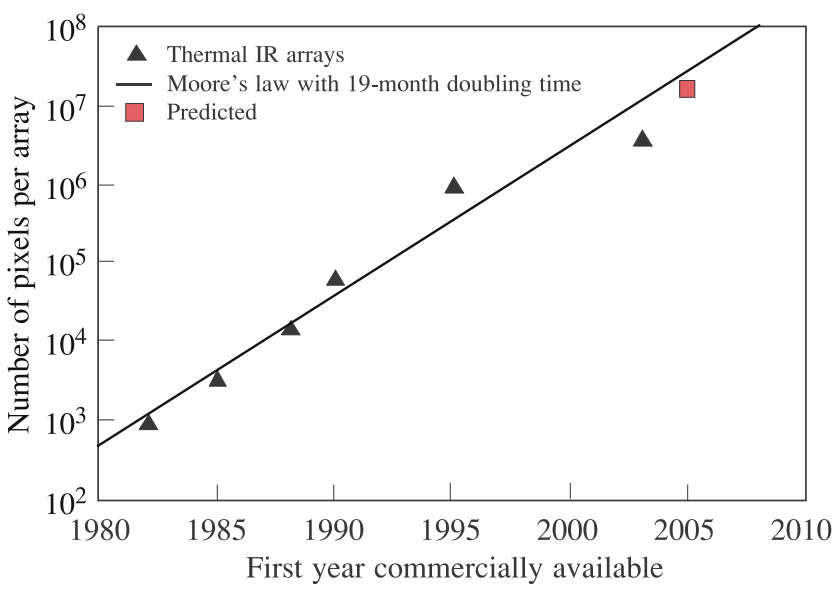

Fig. 1. The number of pixels on an infrared array has been growing exponentially, in accordance with Moore's law for 25 years with a doubling time of approximately 19 months. A $4 \mathrm{~K} \times 4 \mathrm{~K}$ array was predicted for 2005 but is likely at least a year later (after Ref. 15).

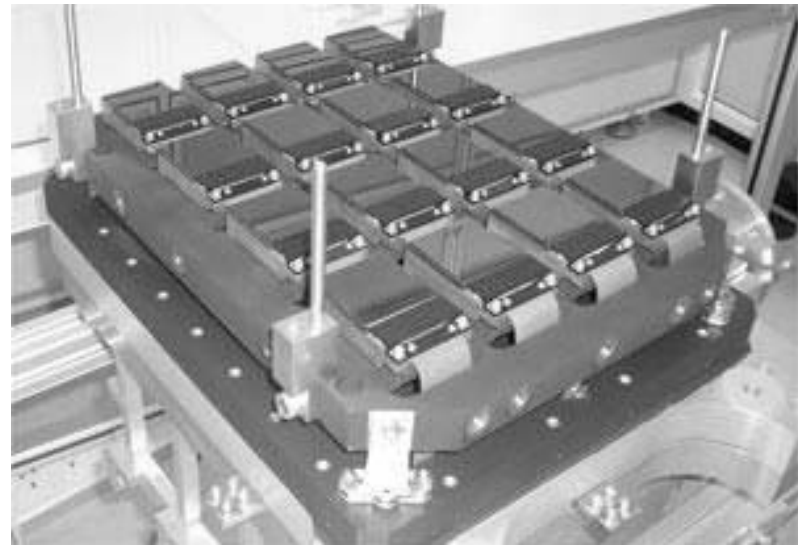

Fig. 2. Sixteen $2048 \times 2048 \mathrm{HgCdTe}$ SCAs were assembled for the VISTA telescope. The SCAs are attached to a precision ground plate that ensures that all pixels are within $12 \mu \mathrm{m}$ of the desired focus. The detectors are ready to be placed in the telescope camera's vacuum chamber and cooled to $72 \mathrm{~K}$ (after Ref. 16).

The definition of third generation IR systems is not particularly well established and not based on any objective criteria. However, it is widely held that third generation detectors are not simply very large arrays with the same features as second generation detectors. In the common understanding, third generation IR systems provide enhanced capabilities like larger number of pixels, higher frame rates, better thermal resolution as well as multicolour functionality and other on-chip functions. According to Reago et al. [17], the third generation is defined in order to maintain the current advantage enjoyed by U.S. and allied armed forces. This class of devices includes both cooled and uncooled FPAs [2,5]:

- high performance, high resolution cooled imagers having two- or three-colour bands,

- medium- to high-performance uncooled imagers,

- very low cost, expendable uncooled imagers.

Many challenges are forced IR community in developing third generation IR imagers including noise equivalent difference temperature (NEDT), pixel and chip size issues, uniformity, identification and detection ranges, etc. Here, we concentrate on advantages and disadvantages of competitive technologies of third generation photon detectors including $\mathrm{HgCdTe}$ photodiodes, $\mathrm{AlGaAs} / \mathrm{GaAs}$ quantum well photodetectors and type II InAs/GaInSb superlattices. Special attention is devoted to material uniformity and NEDT considerations.

\subsection{Noise equivalent difference temperature}

For infrared FPAs, the relevant figure of merit is the noise equivalent temperature difference (NEDT). This parameter represents the temperature change, for incident radiation, that gives an output signal equal to the rms noise level. $N E D T$ is defined

$$
N E D T=\frac{V_{n}(\partial T / \partial Q)}{\left(\partial V_{s} / \partial Q\right)}=V_{n} \frac{\Delta T}{\Delta V_{s}},
$$


where $V_{n}$ is the rms noise and $\Delta V_{s}$ is the signal measured for the temperature difference $\Delta T$. It can be shown that [18]

$$
N E D T=\left(\tau C \eta_{B L I P} \sqrt{N_{w}}\right)^{-1}
$$

where $\tau$ is the optics transmission spectrum and $C$ is the thermal contrast. $N_{w}$ is the number of photogenerated carriers integrated for one integration time, $t_{\text {int }}$, and $Q_{B}$ is the photon flux density incident on the detector area $A_{d}$

$$
N_{w}=\eta A_{d} t_{\text {int }} Q_{B} .
$$

The thermal contrast is the ratio of the derivative of spectral photon incidence to the spectral photon incidence

$$
C=\frac{\partial Q / \partial T}{Q}
$$

The contrast in the MWIR bands at $300 \mathrm{~K}$ is $3.5-4$ percent compared to 1.6 percent for the LWIR band. Percentage of BLIP, $\eta_{B L I P}$, is simply the ratio of photon noise to composite FPA noise [18]

$$
\eta_{B L I P}=\left(\frac{N_{\text {photon }}^{2}}{N_{\text {photon }}^{2}+N_{F P A}^{2}}\right)
$$

It results from the above formulas that the charge handling capacity of the readout, the integration time linked to the frame time, and dark current of the sensitive material becomes the major issues of IR FPAs. The NEDT is inversely proportional to the square root of the integrated charge and therefore the greater the charge, the higher the performance.

The distinction between integration time and the FPA's frame time must be noted. At high backgrounds it is often impossible to handle the large amount of carriers generated over frame time compatible with standard video rates. Off-FPA frame integration can be used to attain a level of sensor sensitivity that is commensurate with the detector-limited $D^{*}$ and not the charge-handling-limited $D^{*}$. Even though the detectivity of LWIR detectors is background limited, the readouts of today can collect only about $1 \%$ of the charge in unit cell from a flux of $10^{16}-10^{17}$ photons $/ \mathrm{cm}^{2} \mathrm{~s}$. Unit cell capacitors fill up in about $100 \mu \mathrm{s}$, while the frame time is on the order of $10 \mathrm{~ms}$.

The well charge capacity is the maximum amount of charge that can be stored on the storage capacitor of each cell. The size of the unit cell is limited to the dimensions of the detector element in the array. Usually, it is assumed that the integration time is such that readout node capacity kept half full. For a $30 \times 30 \mu \mathrm{m}^{2}$ pixel size, the storage capacities are limited to 1 to $5 \times 10^{7}$ electrons. For the LWIR $\mathrm{HgCdTe}$ FPAs, the integration time is usually below $100 \mu$ s. Since the noise power bandwidth $\Delta f=1 / 2 t_{i n t}$, a small integration time causes extra noise in integration.

Current readout technology is based upon CMOS circuitry that has benefited from dramatic and continuing progress in miniaturizing circuit dimensions. Second gen-

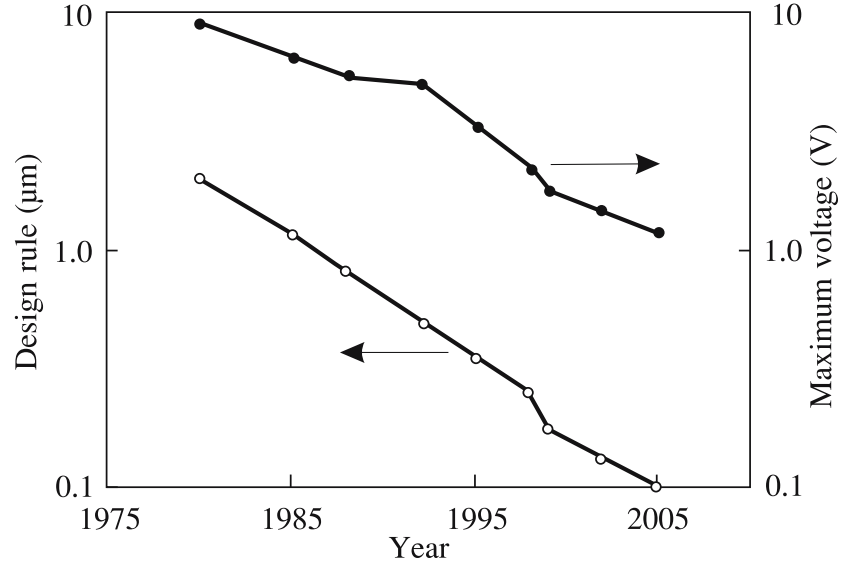

Fig. 3. Trends for design rule minimum dimensions and maximum bias voltage of silicon foundry requirements (after Ref. 2).

eration imagers provide NEDT of about $20-30 \mathrm{mK}$ with $f / 2$ optics. A goal of third-generation imagers is to achieve sensitivity improvement corresponding to NEDT of about 1 $\mathrm{mK}$. It results from Eq. (2) that in a $300 \mathrm{~K}$ scene in LWIR region with thermal contrast of 0.02 , the required charge storage capacity is above $10^{9}$ electrons. This high chargestorage density within the small pixel dimensions cannot be obtained with standard CMOS capacitors [2]. Although the reduced oxide thickness of submicrometer design rules gives large capacitance per unit area, the reduced bias voltage, as illustrated in Fig. 3, largely cancels any improvement in charge storage density.

To provide an opportunity to significantly increase both the charge storage capacity and the dynamic range, the vertically-integrated sensor array (VISA) program has been sponsored by DARPA $[19,20]$. The approach being developed builds on the traditional "hybrid" structure of a detector with a 2-D array of indium-bump interconnects to silicon readout. VISA allows additional layers of silicon processing chips to be connected below the readout to provide more complex functionality. It will allow the use of smaller and multicolour detectors without compromising storage capacity. Signal-to-noise rations will increase for multicolour focal plane arrays. This will permit LWIR focal planes to improve the sensitivity by a factor of ten.

\subsection{Uniformity}

The nonuniformity value is usually calculated using the standard deviation over mean, counting the number of operable pixels in an array. For a system operating in the LWIR band, the scene contrast is about $2 \% / \mathrm{K}$ of change in scene temperature. Thus, to obtain a pixel to pixel variation in apparent temperature to less than, e.g., $20 \mathrm{mK}$, the nonuniformity in response must be less than $0.04 \%$. This is nearly impossible to obtain it in the uncorrected response of the FPA so; a two-point correction is typically used.

FPA uniformity influences an IR system complexity. The uniformity is important for accurate temperature mea- 


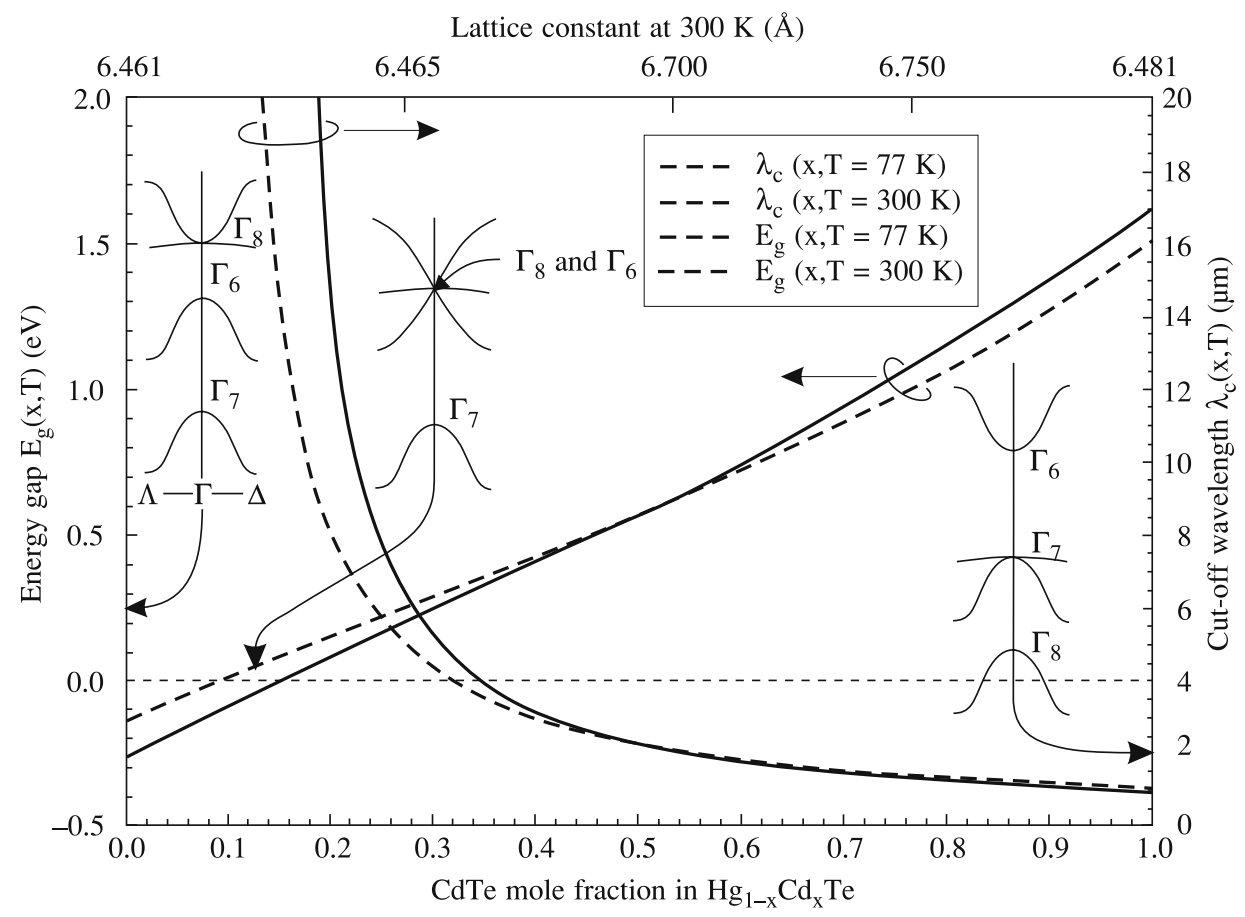

Fig. 4. The bandgap structure of $\mathrm{Hg}_{1-\mathrm{x}} \mathrm{Cd}_{\mathrm{x}} \mathrm{Te}$ near the $\Gamma$-point for three different values of the forbidden energy gap. The energy bandgap is defined at the difference between the $\Gamma_{6}$ and $\Gamma_{8}$ band extrema at $\Gamma=0$.

Table 1. Cutoff wavelength for $x$ variations of $0.2 \%$ and the corresponding cutoff wavelength shift for $\mathrm{Hg}_{1-\mathrm{x}} \mathrm{Cd}_{\mathrm{x}} \mathrm{Te}$.

\begin{tabular}{cccc}
\hline Composition $x$ & $\begin{array}{c}\text { Cutoff wavelength } \\
\lambda_{c}(\mu \mathrm{m})\end{array}$ & $\begin{array}{c}\text { Temperature } T \\
(\mathrm{~K})\end{array}$ & $\begin{array}{c}\text { Uncertainty } \Delta \lambda_{c} \\
(\mu \mathrm{m})\end{array}$ \\
\hline 0.395 & 3 & 77 & 0.023 \\
0.295 & 5 & 77 & 0.064 \\
0.210 & 10 & 77 & 0.260 \\
0.196 & 14 & 77 & 0.510 \\
\hline
\end{tabular}

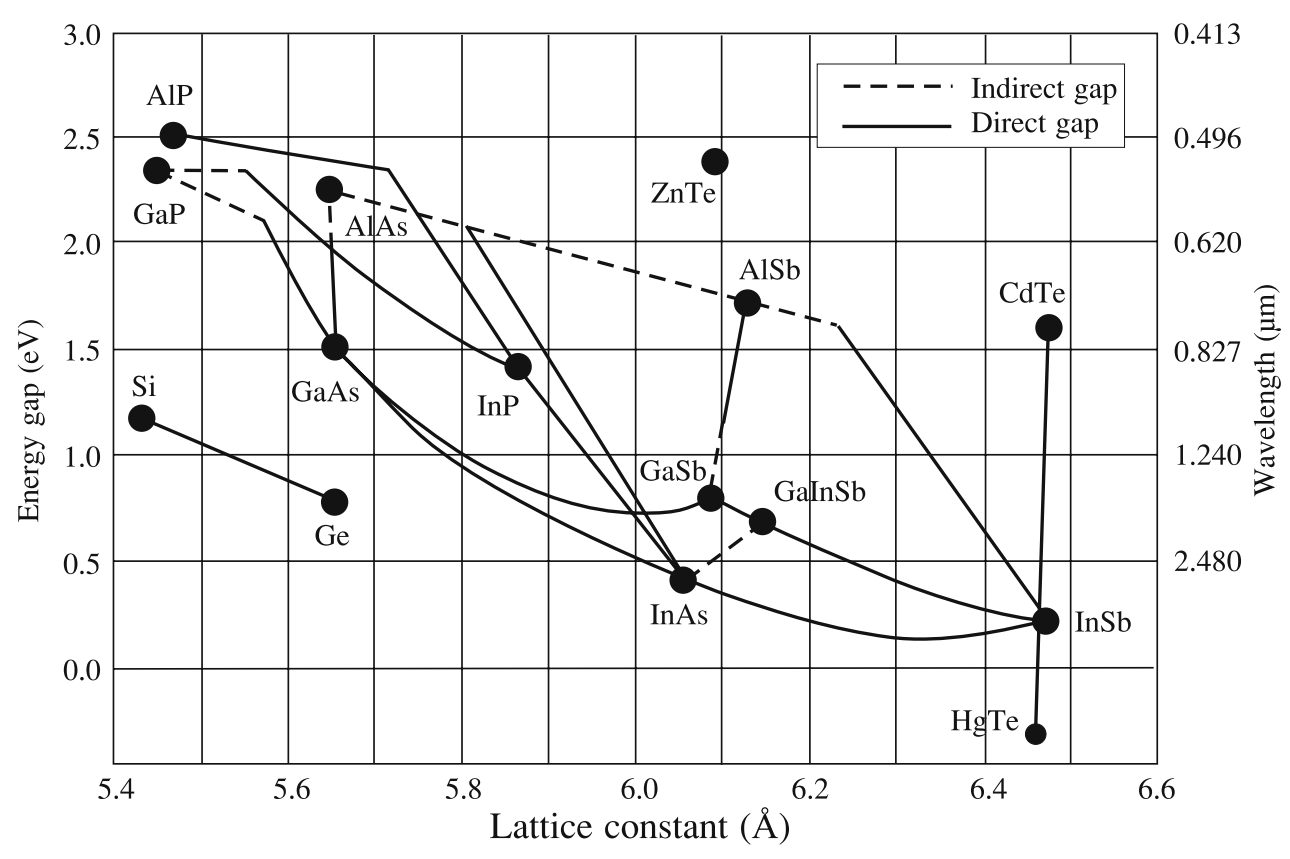

Fig. 5. Composition and wavelength diagram of Sb-based III-V material systems. 
surements, background subtraction, and threshold testing. Nonuniformities require elaboration of compensation algorithms to correct the image and by consuming a number of analogue-to-digital bits they also reduce the system dynamic range.

Figure 4 plots the energy band gap, $E_{g}(x, T)$, for $\mathrm{Hg}_{1-\mathrm{x}} \mathrm{Cd}_{\mathrm{x}} \mathrm{Te}$ versus alloy composition $x$ for temperature of $77 \mathrm{~K}$. Also plotted is the cutoff wavelength $\lambda_{c}(x, T)$, defined as that wavelength at which the response has dropped to $50 \%$ of its peak value. Table 1 shows uncertainty in cutoff wavelength for $x$ variations of $0.2 \%$. For short wavelength IR $(\approx 3 \mu \mathrm{m})$ and MWIR $(\approx 5 \mu \mathrm{m})$ materials, the variation in cut-off wavelength is not large. However, the nonuniformity is a serious problem in the case of LWIR HgCdTe detectors. The variation of $x$ across the $\mathrm{Hg}_{1-\mathrm{x}} \mathrm{Cd}_{\mathrm{x}}$ Te wafer causes much larger spectral nonuniformity; e.g., at $77 \mathrm{~K}$, a variation of $\Delta x=0.2 \%$ gives a $\Delta \lambda_{c}=0.064 \mu \mathrm{m}$ at $\lambda_{c}=5 \mu \mathrm{m}$, but $\Delta \lambda_{c}=0.51 \mu \mathrm{m}$ at $14 \mu \mathrm{m}$ ) which cannot be fully corrected by the two or three point corrections [7]. Therefore required composition control is much more stringent for LWIR than for MWIR. For applications that require operation in the LWIR band as well as two-colour LWIR/VLWIR bands most probably $\mathrm{HgCdTe}$ will not be the optimal solution.

Alternative candidate for third generation IR detectors is Sb-based III-V material system. This material is mechanically robust and have fairly weak dependence of band gap on composition (see Fig. 5).

\section{Material systems of third generation detectors}

In the wavelength regions of interest, such as MWIR and LWIR three detector technologies that are developing multicolour capability are visited here: $\mathrm{HgCdTe}$, QWIPs and antimonide based type II superlattices.

Both $\mathrm{HgCdTe}$ photodiodes and QWIPs have demonstrated the multicolour capability in the MWIR and LWIR range. Each of these technologies has its advantages and disadvantages. QWIP technology is based on the well-developed $\mathrm{A}^{3} \mathrm{~B}^{5}$ material system, which has a large industrial base with a number of military and commercial applications. $\mathrm{HgCdTe}$ material system is only used for detector applications. Therefore QWIPs are easier to fabricate with high yield, high operability, good uniformity and lower cost. On the other hand, $\mathrm{HgCdTe}$ FPAs have higher quantum efficiency, higher operating temperature and potential for the highest performance. So far, however, the $\mathrm{HgCdTe}$ process yield in molecular beam epitaxy (MBE) of growth complex multiplayer structures, especially those working in the LWIR and VLWIR is low, pixel outage rates high and the material is consequently very expensive.

\subsection{HgCdTe}

$\mathrm{HgCdTe}$ ternary alloy is nearly ideal infrared detector material system. Its position is conditioned by three key features:

- tailorable energy band gap over the $1-30-\mu m$ range,
- large optical coefficients that enable high quantum efficiency, and

- favourable inherent recombination mechanisms that lead to high operating temperature.

These properties are direct consequence of the energy band structure of this zinc-blende semiconductor. Moreover, the specific advantages of $\mathrm{HgCdTe}$ are ability to obtain both low and high carrier concentrations, high mobility of electrons, and low dielectric constant. The extremely small change of lattice constant with composition makes it possible to grow high quality layered and graded gap structures. As a result, $\mathrm{HgCdTe}$ can be used for detectors operated at various modes [photoconductor, photodiode or metal-insulator-semiconductor (MIS) detector].

The unit cell of integrated multi-colour FPAs consists of several co-located detectors, each sensitive to a different spectral band. For example, in the back-illuminated dualband detectors, the photodiode with longer cutoff wavelength is grown epitaxially on top of the photodiode with the short cutoff wavelength. The shorter-cutoff photodiode acts as a long-wavelength-pass filter for the longer-cutoff photodiode.

Both sequential mode and simultaneous mode detectors are fabricated from the multi-layer materials. The simplest two-colour $\mathrm{HgCdTe}$ detector, and the first to be demonstrated, is the bias-selectable n-P-N triple-layer heterojunction (TLHJ), back-to-back photodiode shown in Fig. 6(a) (capital letter means wider band gap structure). The n-type base absorbing regions are deliberately doped with indium at a level of about $(1-3) \times 10^{15} \mathrm{~cm}^{-3}$. A critical step in device formation is connected with the in situ p-type As-doped layer with good structural and electrical properties to prevent internal gain from generating spectral crosstalk. The band-gap engineering effort consists of increasing the CdTe mole fraction and the effective thickness of the p-type layer to suppress out-off-band carriers from being collected at the terminal.

The sequential-mode detector has a single indium bump per unit cell that permits sequential bias-selectivity of the spectral bands associated with operating back-to-back photodiodes. When the polarity of the bias voltage applied to the bump contact is positive, the top ( $\mathrm{LW}$ ) photodiode is reverse biased and the bottom (SW) photodiode is forward biased. The SW photocurrent is shunted by the low impedance of the forward-biased SW photodiode, and the only photocurrent to emerge in the external circuit is the LW photocurrent. When the bias voltage polarity is reversed, the situation reverses; only SW photocurrent is available. Switching times within the detector can be relatively short, in the order of microseconds, so detection of slowly changing targets or images can be done by switching rapidly between the MW and LW modes.

One bump contact per unit cell, as for single-colour hybrid FPAs, is the big advantage of the bias-selectable detector. It is compatible with existing silicon readout chips. The problems with the bias selectable device are the following: its construction does not allow independent selection of the 

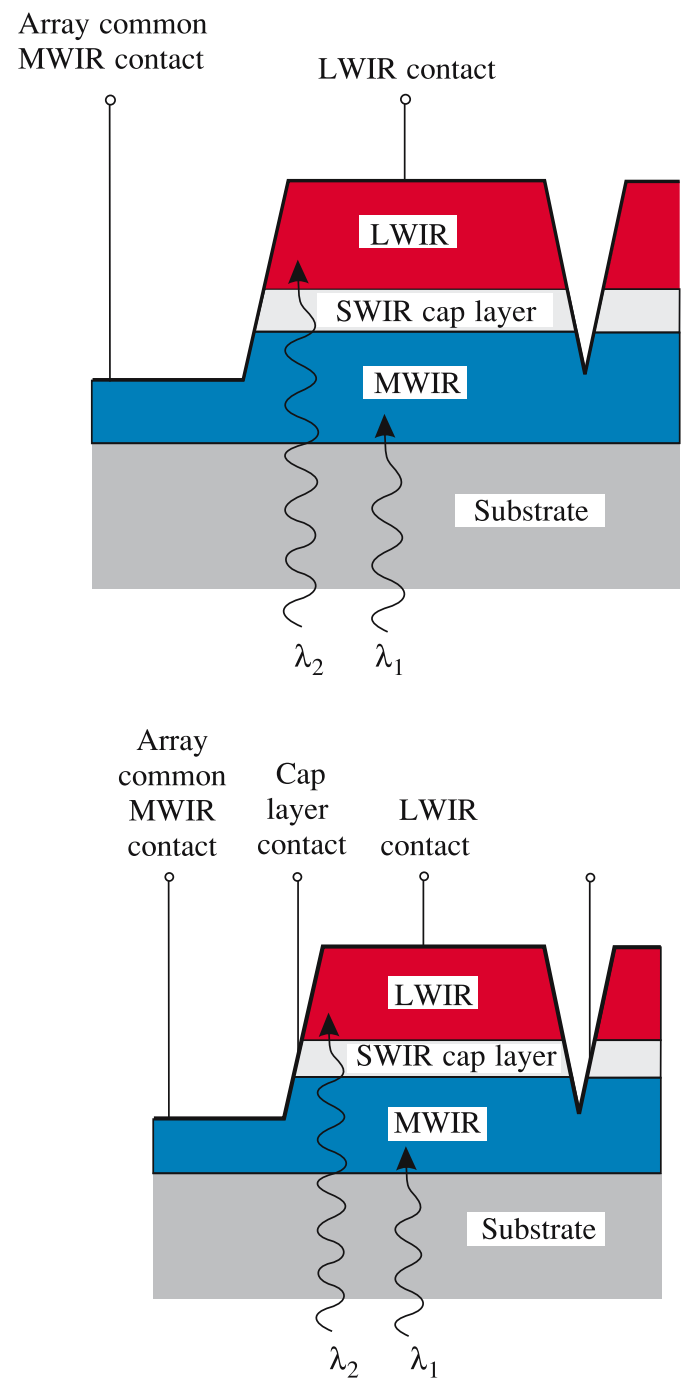

(a)
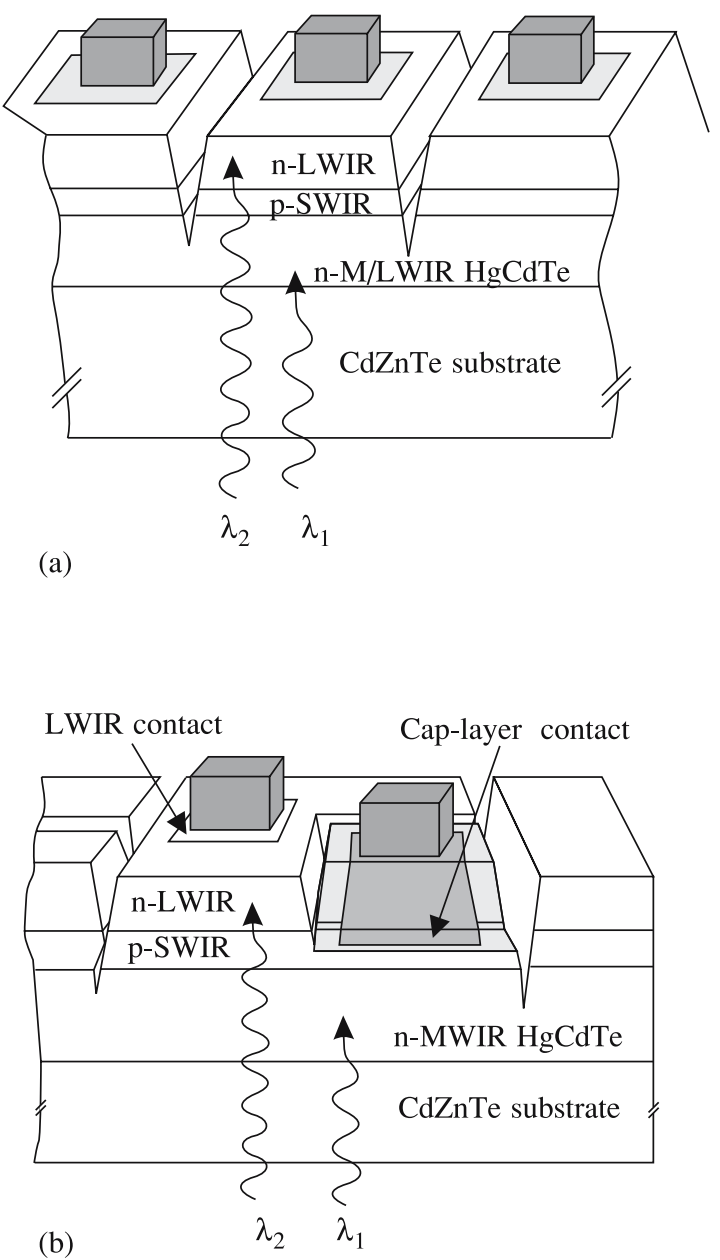

Fig. 6. Two-colour n-P-N detector structure for sequential (a) and simultaneous (b) operating mode.

Table 2. Typical measured performance parameters for single- and dual-colour HgCdTe MWIR and LWIR detector configuration for $256 \times 25630-\mu m$ unit-cell FPAs (after Ref. 21).

\begin{tabular}{|c|c|c|c|c|c|c|c|c|}
\hline \multirow{3}{*}{$\begin{array}{l}256 \times 25630-\mu \mathrm{m} \text { unit-cell } \\
\text { performance parameters }\end{array}$} & \multicolumn{2}{|c|}{ DLHJ single colour } & \multicolumn{6}{|c|}{ TLHJ sequential dual colour } \\
\hline & \multirow{2}{*}{$\begin{array}{l}\text { MWIR } \\
\text { MWIR }\end{array}$} & \multirow{2}{*}{$\frac{\text { LWIR }}{\text { LWIR }}$} & \multicolumn{2}{|c|}{ MWIR/MWIR } & \multicolumn{2}{|c|}{ MWIR/LWIR } & \multicolumn{2}{|c|}{ LWIR/LWIR } \\
\hline & & & Band 1 & Band 2 & Bnad 1 & Band 2 & Band 1 & Band 2 \\
\hline 78-K cutoff $(\mu \mathrm{m})$ & 5 & 10 & 4 & 5 & 5 & 10 & 8 & 10 \\
\hline Operating temperature $(\mathrm{K})$ & 78 & 78 & 120 & 120 & 70 & 70 & 70 & 70 \\
\hline Cross talk $(\%)$ & - & - & $<5$ & $<10$ & $<5$ & $<10$ & $<5$ & $<10$ \\
\hline Quantum efficiency (\%) & $>70$ & $>70$ & $>0$ & $>65$ & $>70$ & $>50$ & $>70$ & $>50$ \\
\hline $\mathrm{R}_{0} \mathrm{~A}$, zero FOV $\left(\Omega \mathrm{cm}^{2}\right)$ & $>1 \times 10^{7}$ & $>500$ & $6 \times 10^{5}$ & $2 \times 10^{5}$ & $1 \times 10^{6}$ & $2 \times 10^{2}$ & $5 \times 10^{4}$ & $5 \times 10^{2}$ \\
\hline $\mathrm{R}_{\mathrm{r}} \mathrm{A}^{*}$, zero FOV $\left(\Omega \mathrm{cm}^{2}\right)$ & - & - & & & & & & \\
\hline Interconnect operability (\%) & $>99.9$ & $>99.9$ & $>99.9$ & $>99.9$ & $>99.9$ & $>99.9$ & $>99.9$ & $>99.9$ \\
\hline Response operability (\%) & $>99$ & $>98$ & $>99$ & $>97$ & $>99$ & $>97$ & $>98$ & $>95$ \\
\hline
\end{tabular}

*resistance area product at nonzero bias 
optimum bias voltage for each photodiode, and there can be substantial MW crosstalk in the LW detector.

Many applications require true simultaneity of detection in the two spectral bands. Simultaneous dual-band detector architectures require an additional electrical contact to an underlying layer in the multijunction structure to both the SW and LW photodiode. An implementation of the simultaneous mode using a second indium bump in the unit cell is shown in Fig. 6(b). The most important distinction is the requirement of a second readout circuit in each unit cell.

Integrated two-colour $\mathrm{HgCdTe}$ technology has been developed for more a decade with a steady progression having a wide variety of pixel size (20 to $61 \mu \mathrm{m})$, array formats $(64 \times 64$ up to $640 \times 480)$ and spectral-band sensitivity (MWIR/MWIR, MWIR/LWIR and LWIR/LWIR). Test structures indicate that the separate photodiodes in a twocolour detector perform exactly as single-colour detectors in terms of achievable $R_{0} A$ product variation with wavelength at a given temperature (see Table 2).

The best performing dual-colour FPAs being produced at Reytheon Vision Systems exhibit out-of-band cross talk below $10 \%, 99.9 \%$ interconnect operability, and $99 \%$ response operability that is comparable to state-of-the-art, single-colour technology. It is predicted that ongoing development of material growth and fabrication process will translate to further improvements in dual-colour FPA performance.

Recently, Raytheon Vision Systems has developed two-colour, large-format infrared FPAs to support the US Army's third generation FLIR systems [22]. RVS has produced $640 \times 480$ two-colour FPAs with a $20-\mu \mathrm{m}$ pixel pitch. Work is also underway to demonstrate a $1280 \times 720$ two-colour FPA. High-quality MWIR/LWIR 640×480 FPAs have cutoffs ranging to $11 \mu \mathrm{m}$ at $78 \mathrm{~K}$. These FPAs have demonstrated excellent sensitivity and pixel operabilities exceeding $99 \%$. NETDs less than $25 \mathrm{mK}$ at $\mathrm{f} / 5$ have been demonstrated for both bands operating simultaneously.

\subsection{QWIP}

An alternative hybrid detector for the middle and long wavelength IR region is the quantum well infrared photoconductors (QWIPs). These high impedance detectors are built from alternating thin layers (superlattices) of GaAs and AlGaAs. Despite large research and development efforts, large photovoltaic LWIR HgCdTe FPAs remain expensive, primarily because of the low yield of operable arrays. The low yield is due to sensitivity of LWIR $\mathrm{HgCdTe}$ devices to defects and surface leakage, which is a consequence of basic material properties. With respect to $\mathrm{HgCdTe}$ detectors, GaAs/AlGaAs quantum well devices have a number of potential advantages, including the use of standard manufacturing techniques based on mature GaAs growth and processing technologies, highly uniform and well-controlled MBE growth on greater than 6 in. GaAs wafers, high yield and thus low cost, more thermal stability, and extrinsic radiation hardness.

LWIR QWIP cannot compete with $\mathrm{HgCdTe}$ photodiode as the single device, especially at higher temperature operation $(>70 \mathrm{~K})$ due to fundamental limitations associated with intersubband transitions. QWIP detectors have relatively low quantum efficiencies, typically less than $10 \%$. The spectral response band is also narrow for this detector, with a full-width, half-maximum of about $15 \%$. All the QWIP data with cutoff wavelength about $9 \mu \mathrm{m}$ is clustered between $10^{10}$ and $10^{11} \mathrm{cmHz}^{1 / 2} / \mathrm{W}$ at about $77 \mathrm{~K}$ operating temperature. However, the advantage of $\mathrm{HgCdTe}$ is less distinct in temperature range below $50 \mathrm{~K}$ due to the problems involved in an $\mathrm{HgCdTe}$ material (p-type doping, Shockley-Read recombination, trap-assisted tunneling, surface and interface instabilities). A more detailed comparison of both technologies has been given by Tidrow et al. [7] and Rogalski [6,9]. Table 3 compares the essential properties of three types of devices at $77 \mathrm{~K}$.

Even though that QWIP is a photoconductor, several its properties such as high impedance, fast response time, and low power consumption well comply requirements of large FPAs fabrication. The main drawbacks of LWIR QWIP FPA technology are the performance limitation for low integration time applications and low operating temperature. Their main advantages are linked to performance uniformity and to availability of large size arrays. The large industrial infrastructure in III-V materials/device growth, processing, and packaging brought about by the utility of GaAs-based devices in the telecommunications industry

Table 3. Essential properties of LWIR HgCdTe and type II SL photodiodes, and QWIPs at $77 \mathrm{~K}$.

\begin{tabular}{|c|c|c|c|}
\hline Parameter & $\mathrm{HgCdTe}$ & QWIP (n-type) & InAs/GaInSb SL \\
\hline IR absorption & normal incidence & $\begin{array}{l}\mathrm{E}_{\text {optical }} \text { plane of well required } \\
\text { normal incidence: no absorption }\end{array}$ & normal incidence \\
\hline Quantum efficiency & $\geq 70 \%$ & $\leq 10 \%$ & $\approx 30 \%$ \\
\hline Spectral sensitivity & wide-band & narrow-band $(\mathrm{FWHM} \approx 1-2 \mu \mathrm{m})$ & wide-band \\
\hline Optical gain & 1 & $0.2(30-50$ wells $)$ & 1 \\
\hline Thermal generation lifetime & $\approx 1 \mu \mathrm{s}$ & $\approx 10 \mathrm{ps}$ & $\approx 0.1 \mu \mathrm{s}$ \\
\hline$R_{0} A$ product $\left(\lambda_{c}=10 \mu \mathrm{m}\right)$ & $300 \Omega \mathrm{cm}^{2}$ & $10^{4} \Omega \mathrm{cm}^{2}$ & $100 \Omega \mathrm{cm}^{2}$ \\
\hline Detectivity $\left(\lambda_{\mathrm{c}}=10 \mu \mathrm{m}, \mathrm{FOV}=0\right)$ & $2 \times 10^{12} \mathrm{cmHz}^{1 / 2} \mathrm{~W}^{-1}$ & $2 \times 10^{10} \mathrm{cmHz}^{1 / 2} \mathrm{~W}^{-1}$ & $5 \times 10^{11} \mathrm{cmHz}^{1 / 2} \mathrm{~W}^{-1}$ \\
\hline
\end{tabular}


gives QWIPs a potential advantage in productivity and cost. The only known use of $\mathrm{HgCdTe}$, to the date, is for IR detectors.

State of the art QWIP and HgCdTe FPAs provide similar performance figure of merit, because they are predominantly limited by the readout circuits.

It can be shown that NEDT value for charge-limited $\mathrm{HgCdTe}$ photodiodes can be determined by equation [23]

$$
N E D T=\frac{2 k T_{B}^{2} \bar{\lambda}}{h c \sqrt{2 N_{w}}},
$$

where $\bar{\lambda}=\left(\lambda_{1}+\lambda_{2}\right) / 2$ is the average wavelength of the spectral band between $\lambda_{1}$ and $\lambda_{2}$. If one assumes a typical storage capacity of $2 \times 10^{7}$ electrons, $\bar{\lambda}=10 \mu \mathrm{m}$, and $T_{B}=$ $300 \mathrm{~K}$, Eq. (6) yields NEDT of $19.8 \mathrm{mK}$.

The same estimation for QWIP gives [23]

$$
N E D T=\frac{2 k T_{B}^{2} \bar{\lambda}}{h c} \sqrt{\frac{g}{N_{w}}} .
$$

where $g$ is the photoconductive gain.

Comparing Eqs. (6) and (7) one may notice that the value of NEDT in a charge-limited QWIP detectors is better than that of $\mathrm{HgCdTe}$ photodiodes by a factor of $(2 g)^{1 / 2}$ since a reasonable value of $g$ is 0.4 . Assuming the same operation conditions as for $\mathrm{HgCdTe}$ photodiodes, the value of NEDT is $17.7 \mathrm{mK}$. Thus, a low photoconductive gain actually increases the $S / N$ ratio and a QWIP FPA can have a better NEDT than an HgCdTe FPA with a similar storage capacity.

The above deduction was confirmed experimentally by a research group at Fraunhofer IAF. Based on the photovoltaic low-noise four-zone QWIP structure, the Fraunhofer group [24,25] has manufactured a $256 \times 256$ FPA camera operating at $77 \mathrm{~K}$ with the 9 - $\mu \mathrm{m}$ cutoff wavelength. The camera exhibits record-low NEDT values of $7.4 \mathrm{mK}$ with 20 -ms integration time and $5.2 \mathrm{mK}$ with $40 \mathrm{~ms}$. It is the best temperature resolution ever obtained in the LWIR regime.

The very short integration time of LWIR HgCdTe devices of typically below $300 \mu$ s is very useful to freeze a scene with rapidly moving objects. QWIP devices achieve, due to excellent homogeneity and low photoelectrical gain, an even better NEDT, however, the integration time must be 10 to 100 times longer for that, and typically it is 5-20 ms. Decision of the best technology is therefore driven by the specific needs of a system. Even $\mathrm{HgCdTe}$ photodiodes intrinsically exhibit higher performance that the QWIP detectors, QWIP detectors are used for large formats (e.g. $1024 \times 1024$ and larger) with low frame rates and large integration time. Recently, 1 magapixel hybrid MWIR and LWIR QWIPs with 18- $\mu$ m pixel size have been demonstrated with excellent imaging performance [26,27]. The MWIR detector arrays has demonstrated a NEDT of $17 \mathrm{mK}$ at a $95 \mathrm{~K}$ operating temperature with $f / 2.5$ optics at 300 background and the LWIR detector array has demonstrated a NEDT of $13 \mathrm{mK}$ at a $70 \mathrm{~K}$ operating temperature with the some optical and background conditions as the MWIR detector array [27]. This technology can be extended to a $2 \mathrm{~K} \times 2 \mathrm{~K}$ array, but at present the limitation is the readout availability and cost.

Powerful possibilities of QWIP technology are connected with multicolour detection. Four-band FPAs have been demonstrated by stacking different multi-quantum well structures, which are sensitive in 4-6, 8.5-10, 10-12, and $13-15 \mu \mathrm{m}$ bands [28]. The $640 \times 512$ format FPA consists of four $640 \times 128$ pixel areas which are capable of acquiring images in these bands. Four separate detector bands were defined by a deep trench etch process and the unwanted spectral bands were eliminated by a detector short-circuiting process. The unwanted top detectors were electrically shorted by gold-coated reflective 2D etched gratings as shown in Fig. 7.

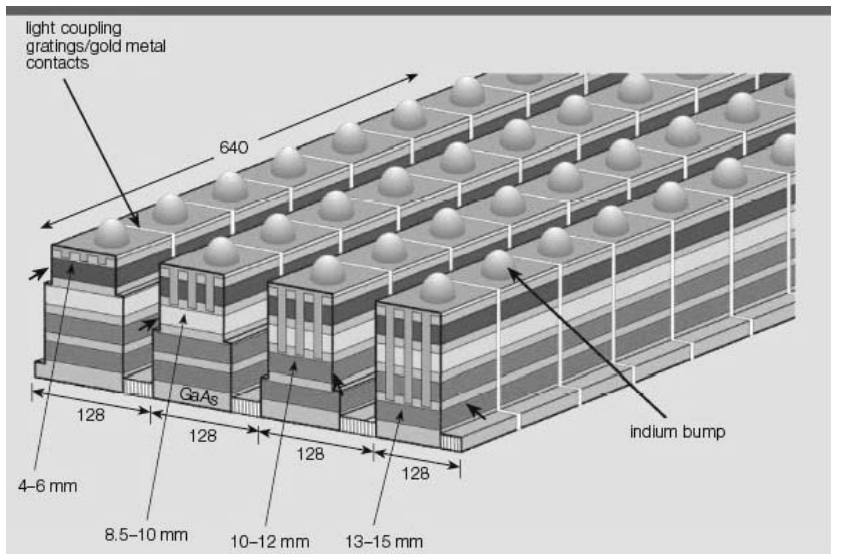

Fig. 7. JPL's four-band QWIP imager, which detects in the 4-6- $\mu \mathrm{m}$, $8.5-10-\mu \mathrm{m}, 10-12-\mu \mathrm{m}$, and $13-15-\mu \mathrm{m}$ spectral bands. The deep groove 2D-periodic grating structure is shown. Each pixel represents a 640128 pixel area of the four-band FPA (after Ref. 28).

\section{InAs/GaInSb as a promising material system for third generation detectors}

InAs/ $\mathrm{Ga}_{1-\mathrm{x}} \mathrm{In}_{\mathrm{x}} \mathrm{Sb}$ (InAs/GaInSb) strained layer superlattices (SLSs) can be considered as an alternative to $\mathrm{HgCdTe}$ and GaAs/AlGaAs IR material system as a candidate for third generation IR detectors. The low quantum efficiency of QWIP's is largely due to fact that the optical transition is forbidden for normal incidence of light. Straylight generated by reflecting gratings is required to achieve reasonable quantum efficiency. On the other hand this straylight degrades the modulation transfer function of QWIP's since some light intensity is guided by the residual substrate into neighbours. In the case of InAs/GaInSb SLS structures, the absorption is strong for normal incidence of light. Consequently, the SLS structures provide high responsivity, as already reached with $\mathrm{HgCdTe}$, without any need for gratings. Further advantages are a photovoltaic operation 
mode, operation at elevated temperatures and well established III-V process technology.

InAs/GaInSb material system is, however, in a very early stage of development. Problems exist in material growth, processing, substrate preparation, and device passivation [11]. Optimization of SL growth is a trade-off between interfaces roughness, with smoother interfaces at higher temperature, and residual background carrier concentrations, which are minimized on the low end of this range. The thin nature of InAs and GaInSb layers $(<8 \mathrm{~nm})$ necessitate low growth rates for control of each layer thickness to within 1 (or 1/2) monolayer (ML). Monolayer fluctuations of the InAs layer thickness can shift the cut-off wavelength by about $\pm 2 \mu \mathrm{m}$ for a 20 - $\mu \mathrm{m}$ designed cut-off. Typical growth rates are less than $1 \mathrm{ML} / \mathrm{s}$ for each layer.

\subsection{Material properties}

The type-II superlattice has staggered band alignment such that the conduction band of the InAs layer is lower than the valence band of InGaSb layer, as shown in Fig. 8. This creates a situation in which the energy band gap of the superlattice can be adjusted to form either a semimetal (for wide InAs and GaInSb layers) or a narrow band gap (for narrow layers) semiconductor material. In the SL, the electrons are mainly located in the InAs layers, whereas holes are confined to the GaInSb layers. This suppresses Auger recombination mechanisms and thereby enhances carrier lifetime. Optical transitions occur spatially indirectly and, thus, the optical matrix element for such transitions is relatively small. The band gap of the SL is determined by the energy difference between the electron miniband $E_{1}$ and the first heavy hole state $H H_{1}$ at the Brillouin zone centre and can be varied continuously in a range between 0 and about $250 \mathrm{meV}$. An example of the wide tunability of the SL is shown in Fig. 8(b).

It has been suggested that InAs/ $\mathrm{Ga}_{1-\mathrm{x}} \mathrm{In}_{\mathrm{x}} \mathrm{Sb}$ SLSs material system can have some advantages over bulk $\mathrm{HgCdTe}$, including lower leakage currents and greater uniformity [30,31]. Electronic properties of SLSs may be superior to those of the $\mathrm{HgCdTe}$ alloy [31]. The effective masses are

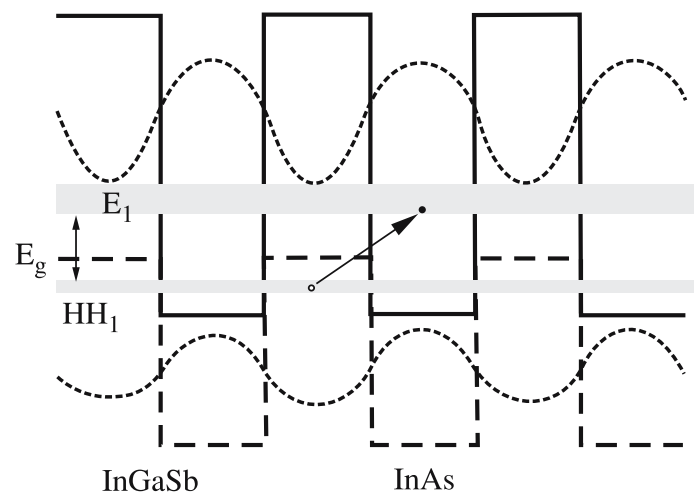

not directly dependent on the band gap energy, as it is the case in a bulk semiconductor [32]. The electron effective mass of InAs/GaInSb SLS is larger $\left(\mathrm{m}^{*} / \mathrm{m}_{\mathrm{o}} \approx 0.02-0.03\right.$, compared to $m^{*} / m_{\mathrm{o}}=0.009$ in $\mathrm{HgCdTe}$ alloy with the same band gap $E_{g} \approx 0.1 \mathrm{eV}$ ) [33]. Thus, diode tunneling currents in the SL can be reduced compared to the $\mathrm{HgCdTe}$ alloy. Although in-plane mobilities drop precipitously for thin wells, electron mobilities approaching $10^{4} \mathrm{~cm}^{2} / \mathrm{Vs}$ have been observed in InAs/GaInSb superlattices with the layers less than $40 \AA$ thick. While mobilities in these SLs are found to be limited by the same interface roughness scattering mechanism, detailed band structure calculations reveal a much weaker dependence on layer thickness, in reasonable agreement with experiment [34].

A consequence of the type II band alignment of InAs/ GaInSb material system is spatial separation of electrons and holes. This is particularly disadvantageous for optical absorption, where a significant overlap of electron and hole wave function is needed. However, a reduction in the electronic confinement can be achieved by growing thinner GaInSb barriers or by introducing more indium into the GaInSb layers leading to optical absorption coefficient comparable to that of $\mathrm{HgCdTe}$.

Theoretical analysis of band-to-band Auger and radiative recombination lifetimes for InAs/GaInSb SLSs showed that in these objects the p-type Auger recombination rates are suppressed by several orders, compared to those of bulk $\mathrm{HgCdTe}$ with similar band-gap $[35,36]$, but n-type materials are less advantageous. However, the promise of Auger suppression has not yet to be observed in practical device material.

Comparison of theoretically calculated and experimentally observed lifetimes at $77 \mathrm{~K}$ for $10 \mu \mathrm{m}$ InAs/GaInSb SLS and $10 \mu \mathrm{m} \mathrm{HgCdTe}$ is presented in Fig. 9. The agreement between theory and experiment for carrier densities above $2 \times 10^{17} \mathrm{~cm}^{-3}$ is good. The discrepancy between both types of results for lower carrier densities is due to Shockley-Read recombination processes having a $\tau \approx 6 \times 10^{-9} \mathrm{~s}$, which has been not taken into account in the calculations. For higher carrier densities, the SL carrier lifetime is two orders of magnitude longer than in $\mathrm{HgCdTe}$, however in

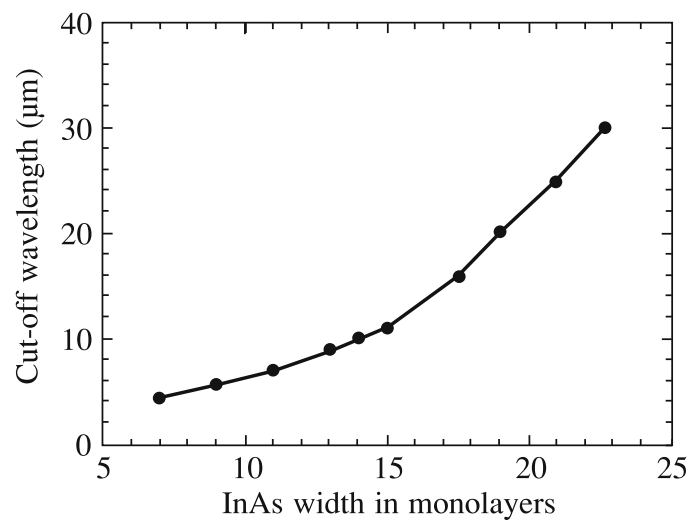

Fig. 8. InAs/GaInSb strained layer superlattice: (a) band edge diagram illustrating the confined electron and hole minibands which form the energy band gap; (b) change in cut-off wavelength with change in one superlattice parameter - InAs layer width (after Ref. 29). 


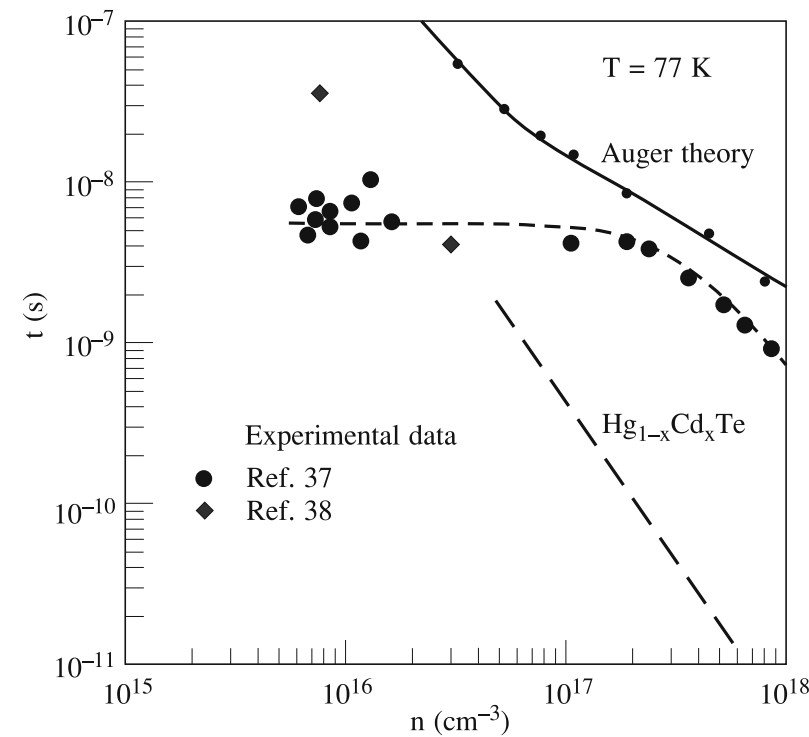

Fig. 9. Comparison of measured and calculated carrier lifetimes of InAs/GaInSb SLS (about $120 \mathrm{eV}$ energy gap) at $77 \mathrm{~K}$ as a function of carrier density. Experimental data are taken from Refs. $37(\bullet)$ and 38 ( ). Theory after Ref. 37.

low doping region (below $10^{15} \mathrm{~cm}^{-3}$, necessary in fabrication high performance p-on-n $\mathrm{HgCdTe}$ photodiodes) experimentally measured carrier lifetime in $\mathrm{HgCdTe}$ is more that two orders of magnitude longer than in SL. Recently published upper experimental data [38] coincide well with $\mathrm{HgCdTe}$ trend-line in the range of lower carrier concentration (see Fig. 9). In general however, the SL carrier lifitime is limited by influence of trap centres located at an energy level of $\sim 1 / 3$ band gap below the effective conduction band edge.

Narrow band gap materials require the doping to be controlled to at least $1 \times 10^{15} \mathrm{~cm}^{-3}$ or below to avoid deleterious high-field tunneling currents across reduced depletion widths at temperature below $77 \mathrm{~K}$. Lifetimes must be increased to enhance carrier diffusion and reduce related dark currents. At present stage of development, the residual doping concentration (both n-type as well as p-type) is typically about $5 \times 10^{15} \mathrm{~cm}^{-3}$ in superlattices grown at substrate temperature ranging from $360^{\circ} \mathrm{C}$ to $440^{\circ} \mathrm{C}$ [11]. Low to mid $10^{15} \mathrm{~cm}^{-3}$ residual carrier concentrations are the best that have been achieved so far.

\subsection{Superlattice photodiodes}

High performance InAs/GaInSb SL photovoltaic is predicted by the theoretical promise of longer intrinsic lifetimes due to the suppression of Auger recombination mechanism. Figure 10 compares theoretical temperature dependence of detectivity of two types of $\mathrm{HgCdTe}$ photodiodes and InAs/InGaSb SL photovoltaic detectors operating at $11 \mu \mathrm{m}$. The curves calculated for two InAs/GaInSb SLs (39.8 $\mathrm{InAs} / 15 \AA \mathrm{Ga}_{0.6} \mathrm{In}_{0.4} \mathrm{Sb}$ and $41 \AA \AA \mathrm{InAs} /$ $25 \AA \mathrm{Ga}_{0.75} \mathrm{In}_{0.25} \mathrm{Sb}$ ) are taken from Ref. 39. As Fig. 10 shows, the ultimate detectivity of $\mathrm{HgCdTe}$ photodiodes

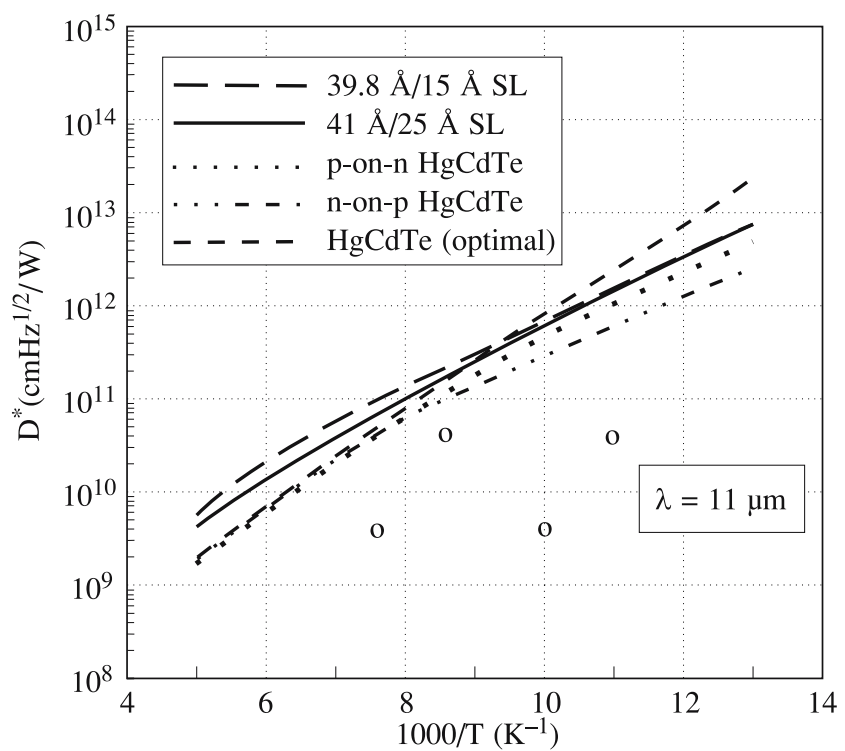

Fig. 10. Calculated detectivity of three types $\mathrm{HgCdTe}$ photodiodes and InAs/InGaSb SL photovoltaic detectors operated at $11 \mu \mathrm{m}$ as a function of temperature. The base-region limited detectivities are calculated for $\mathrm{HgCdTe}$ photodiodes assuming: $\eta=1, t=10 \mu \mathrm{m}, \mathrm{N}_{\mathrm{a}}$ $=5 \times 10^{15} \mathrm{~cm}^{-3}$ for n-on-p structures; $\eta=1, t=10 \mu \mathrm{m}, N_{d}=3 \times 10^{14}$ $\mathrm{cm}^{-3}$ for p-on-n structures; and $\eta=1, t=10 \mu \mathrm{m}$ for photodiode with optimal doping in the base region. The curves calculated for two InAs/InGaSb SLs (39.8 $\AA$ InAs/15 $\AA \mathrm{In}_{0.4} \mathrm{Ga}_{0,6} \mathrm{Sb}$ and $41 \AA$ InAs/25 $\mathrm{In}_{0.25} \mathrm{Ga}_{0,75} \mathrm{Sb}$ ) are taken from Ref. 39 (after Refs. 40 ).

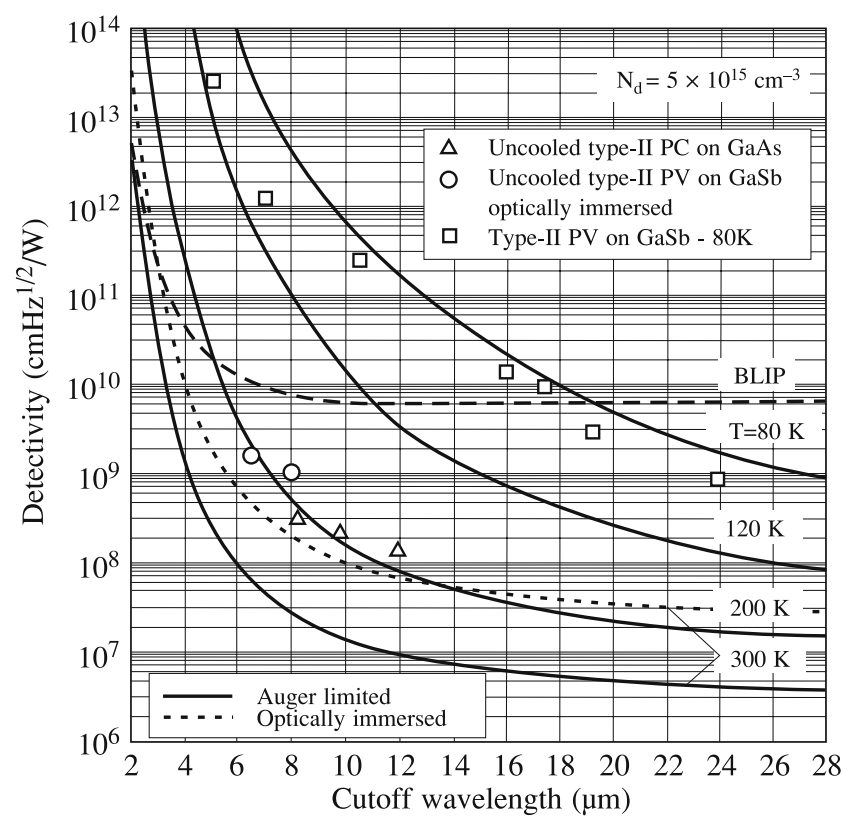

Fig. 11. Calculated performance of Auger generation-recombination limited $\mathrm{HgCdTe}$ photodetectors as a function of wavelength and temperature of operation. BLIP detectivity has been calculated for $2 \pi \mathrm{FOV}$, background temperature $T_{B L I P}=300 \mathrm{~K}$, and quantum efficiency $\eta=1$. The experimental data is taken for cooled and uncooled type II detectors at Center for Quantum Devices, Northwestern University. The calculations have been performed for doping level equal $N_{d}=5 \times 10^{15} \mathrm{~cm}^{-3}$. 
with optimally doped base region are comparable with that of $\mathrm{InAs} / \mathrm{InGaSb} \mathrm{SLs}$ in the temperature region between $300 \mathrm{~K}$ and $77 \mathrm{~K}$. High performance of InAs/GaInSb SLSs detectors (also at higher operating temperatures) is a result of long Auger carrier lifetimes caused by the splitting of the light-hole and heavy-hole bands.

The Auger mechanism is likely to impose fundamental limitations to the LWIR $\mathrm{HgCdTe}$ detector performance. Figure 11 compares the calculated detectivity of Auger generation-recombination limited $\mathrm{HgCdTe}$ photodetectors as a function of wavelength and temperature of operation with the experimental data of cooled and uncooled type II detectors at Centre for Quantum Devices, Northwestern University. The calculations have been performed for doping level equal $N_{d}=5 \times 10^{15} \mathrm{~cm}^{-3}$. These results indicate that the type II superlattice is a good candidate for infrared detectors operating in the spectral range from the mid wave to the very long wave infrared. In addition this type superlattice is an excellent candidate for uncooled applications. The measured detectivity is more than $10^{8} \mathrm{cmHz}^{1 / 2} / \mathrm{W}$ at $10.6 \mu \mathrm{m}$ at room temperature, which is higher than the commercially available uncooled $\mathrm{HgCdTe}$ detectors at similar wavelength [41].

Considering the $n$-on- $p$ and the $p$-on- $n$ structures, the n-on-p geometry (n-type cap contact layer on a p-type absorber) has been theoretically shown to be desirable based on favourable Auger lifetimes and superior minority carrier transport properties associated with lightly doped p-type SLS.

Superlattice photodiodes are based p-i-n structures with an unintentionally doped, intrinsic region between the heavily doped contact portions of the device. The layers are grown by $\mathrm{MBE}$ at substrate temperatures around $400^{\circ} \mathrm{C}$ on undoped (001) oriented two-inch GaSb substrates. Despite the relatively low absorption coefficients, GaSb substrates require thinning the thickness below $25 \mu \mathrm{m}$ in order to transmit appreciable IR radiation [42]. Since the GaSb substrates and buffer layers are intrinsically p-type, the p-type contact layer, intentionally doped with beryllium at an acceptor concentration of $1 \times 10^{18}$ atoms $/ \mathrm{cm}^{3}$, is grown first (see Fig. 12). These acceptor doped SL layers are followed by a 1 to $2 \mu \mathrm{m}$ thick, nominally undoped, superlattice re-

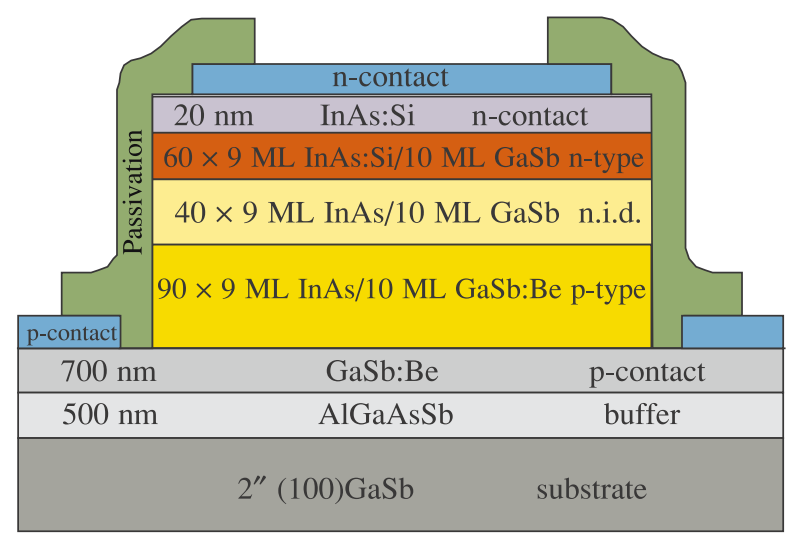

(a) gion. The width of the intrinsic region does very in the designs. The width used should be correlated to the carrier diffusion lengths for improved performance. The upper of the SL stack is the InAs layers doped with silicon $\left(1 \times 10^{17}\right.$ to $\left.1 \times 10^{18} \mathrm{~cm}^{-3}\right)$ and is typically $0.5 \mu \mathrm{m}$ thick. The top of the SL stack is then capped with an InAs:Si $\left(n \approx 10^{18} \mathrm{~cm}^{-3}\right)$ layer to provide good ohmic contact.

Because the energy band gaps on the order of several hundred meV are needed to cover the mid-infrared, only the binary/binary versions of the InAs/GaSb SL are used [see Fig. 12(a)]. The layers needed are already so thin that there is no benefit to using GaInSb alloys.

To approach cut-off wavelengths in the 8-to-12 $\mu \mathrm{m}$ wavelength range the InAs/GaInSb short period superlattice $\mathrm{p}$-i-n photodiodes, with the indium molar fraction in the ternary GaInSb layers close to $20 \%$, are fabricated. A cross section scheme of a completely processed mesa detector is presented in Fig. 12(b). MBE growth started with a $500 \mathrm{~nm}$ thick $\mathrm{Al}_{0.5} \mathrm{Ga}_{0.5} \mathrm{As}_{0.04} \mathrm{Sb}_{0.96}$ lattice matched buffer layer followed by a p-type contact layer comprised of 700 $\mathrm{nm} \mathrm{GaSb}: \mathrm{Be}\left(3 \times 10^{18} \mathrm{~cm}^{-3}\right)$. The active region contains 190 periods of an 8.6 ML InAs/5.0 ML $\mathrm{Ga}_{0.75} \operatorname{In}_{0.25} \mathrm{Sb}$ superlattice structure. The active region is terminated by a $30 \mathrm{~nm}$ $\mathrm{GaSb}$ cap layer. The lower 90 periods of the SL were p-doped with $1 \times 10^{17} \mathrm{~cm}^{-3}$ Be in the $\mathrm{Ga}_{0.75} \mathrm{In}_{0.25} \mathrm{Sb}$ layers. The next 40 SL-periods were not intentionally doped followed by 60 periods with an n-doping of $1 \times 10^{17} \mathrm{~cm}^{-3} \mathrm{Si}$ in the InAs layers.

The main technological challenge for the fabrication of small area size photodiodes is the occurrence of surface leakage currents mainly due to tunneling electrons. Besides efficient suppression of surface leakage currents, a passivation layer suitable for production purposes must withstand various treatments occurring during the subsequent processing of the device. Several materials and processes have been explored. Some of the more prominent thin films studied have been silicon nitride, silicon oxides, ammonium sulfide and most recently, aluminium gallium antimonide alloys [43]. It appears that the reproducibility and long-term stability achieved by the dielectric passivation layer is not sufficient for photodiodes in the LWIR range.

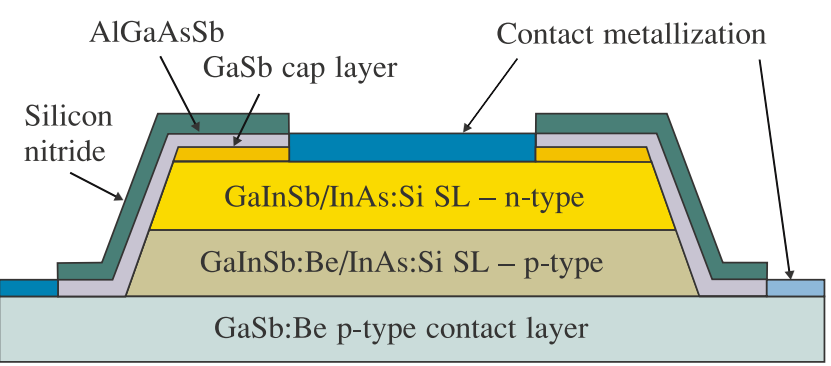

(b)

Fig. 12. Cross section schematic of p-i-n InAs/GaInSb superlattice photodiodes for the MWIR (a) and LWIR (b) spectral regions (after Ref. 44). 


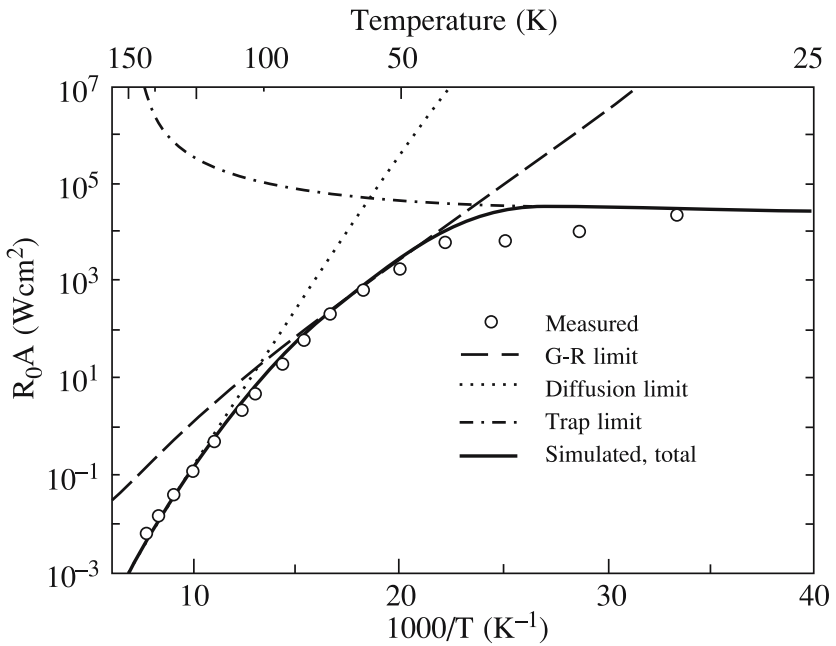

Fig. 13. Experimental data and theoretical prediction of the $R_{0} A$ product as a function of temperature for InAs/GaInSb photodiode with $11-\mu \mathrm{m}$ cut-off wavelength. The activated trap density is taken as a constant $\left(1 \times 10^{12} \mathrm{~cm}^{-3}\right)$ in the simulation over the whole temperature range (after Ref. 38).

Therefore, Rehm et al. have chosen and demonstrated the impressive results achieved with lattice matched AlGaAsSb overgrowth by MBE on etched mesas [44].

The best detector characteristics reported to date are from the recent works at the Fraunhofer Institute in Freiburg $[12,13,44]$. The performance of LWIR photodiodes in the high temperature range is limited by diffusion process. For example, Fig. 13 shows the experimental data and theoretical prediction of the $\mathrm{R}_{0} \mathrm{~A}$ product as a function of temperature for $\mathrm{InAs} / \mathrm{GaInSb}$ photodiode with $11-\mu \mathrm{m}$ cutoff wavelength. The photodiodes are depletion region (generation-recombination) limited in temperature range between $80 \mathrm{~K}$ and $50 \mathrm{~K}$. The trap-assisted tunneling is dominant only at low temperature $(<50 \mathrm{~K})$ with almost constant activation trap density $\left(1 \times 10^{12} \mathrm{~cm}^{-3}\right)$. Assuming

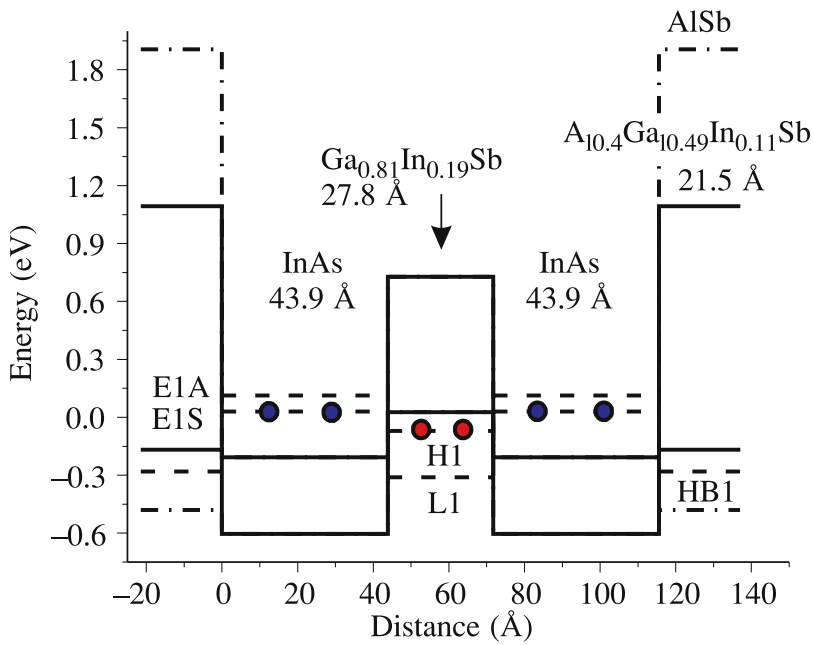

Fig. 14. High quantum efficiency WSL heterostructure used for "i-region" absorber layer. AlSb barrier layers are indicated by dashed lines for comparison with QBLs (after Ref. 45). identical material quality with the same activated trap density, the $R_{0} A$ values exceeding $100 \Omega \mathrm{cm}^{2}$ even with a cut-off wavelength of $14 \mu \mathrm{m}$ can be achieved.

Optimization of the SL photodiode architectures is still an open area. Since some of the device design parameters depend on material properties, like carrier lifetime and diffusion lengths, these properties are still being improved. For example, recently Aifer et al. [45] have reported Wstructured type II superlattice LWIR photodiodes with $\mathrm{R}_{0} \mathrm{~A}$ values comparable for state-of-the-art $\mathrm{HgCdTe}$. These structures initially developed to increase the gain in MWIR lasers, are now showing promise as LWIR and VLWIR photodiodes materials. In this design illustrated in Fig. 14, the AlSb barriers are replaced with shallower $\mathrm{Al}_{0.4} \mathrm{Ga}_{0.49} \mathrm{In}_{0.11} \mathrm{Sb}$ quaternary barrier layers (QBL) that have a much smaller conduction band-offset with respect to InAs, resulting in higher electron mobility, with a miniband width of about $35 \mathrm{meV}$, compared to $20 \mathrm{meV}$ for AlSb barrier layers. The QBL also uses 60\% less Al, which improves material quality, since the optimal QBL growth temperature is much closer than that of $\mathrm{AlSb}\left(\sim 500^{\circ} \mathrm{C}\right)$ to that of the InAs and InGaSb layers around $430^{\circ} \mathrm{C}$. In such structure two InAs "electron-wells" are located on either side of an InGaSb "hole-well" and are bound on either side by AlGaInSb "barrier" layers. The barriers confine the electron wavefunctions symmetrically about the hole-well, increasing the electron-hole overlap while nearly localizing the wavefunctions. The resulting quasi-dimensional densities of states give the WSL its characteristically strong absorption near band-edge. However, care is taken to not fully localize the wavefunctions, since an electron mini-band is required to allow vertical transport of the photo-excited minority carriers.

The new design W-structured type II SL photodiodes employ a graded band-gap p-i-n design which has resulted in an order of magnitude improvement in dark current per-

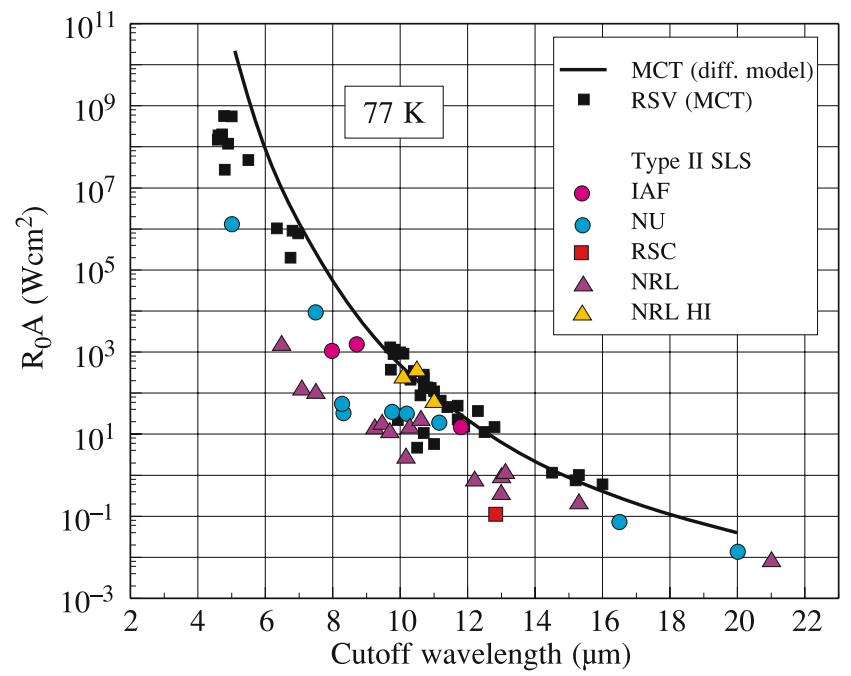

Fig. 15. Dependence of the $R_{O} A$ product of InAs/GaInSb SLS photodiodes on cutoff wavelength compared to theoretical and experimental trendlines for comparable $\mathrm{HgCdTe}$ photodiodes at 77 K (after Ref. 45). 
formance, with $R_{0} A=174 \Omega \mathrm{cm}^{2}$ at $80 \mathrm{~K}$, and excess of $300 \Omega \mathrm{cm}^{2}$ at $77 \mathrm{~K}$, for devices with a $10.5 \mu \mathrm{m}$ cutoff wavelength.

Figure 15 compares the $R_{0} A$ values of InAs/GaInSb SL and $\mathrm{HgCdTe}$ photodiodes in the long wavelength spectral range. The solid line denotes the theoretical diffusion limited performance of p-type $\mathrm{HgCdTe}$ material. As it can be seen in the figure, the most recent photodiode results for SL devices rival that of practical $\mathrm{HgCdTe}$ devices, indicating substantial improvement has been achieved in SL detector development.

\subsection{Focal plane arrays}

The presented results indicate that fundamental material issues of InAs/GaInSb SLs fulfil practical realization of high performance FPAs. As grown material has exhibited a moderate residual doping about $5 \times 10^{15} \mathrm{~cm}^{-3}$. Up till now non-optimized carrier lifetimes have been observed and at desirably low carrier concentrations is limited by Shockley- Read recombination mechanism.

Apart from these limitations, first $256 \times 256$ SL MWIR detectors have been hybridized [12]. The cut-off wavelength of this detector is $5.3 \mu \mathrm{m}$. Excellent NEDT value of approximately $10 \mathrm{mK}$ measured with $f / 2$ optics and integration time $\tau_{\text {int }}=5 \mathrm{~ms}$ has been presented. Tests with reduced time down to $1 \mathrm{~ms}$ show that the NEDT scales inversely proportional to the square root of the integration time [see Eq. (2)]. It means that even for short integration time the detectors are background limited.

Very important feature of InAs/GaInSb FPAs is their high uniformity [12]. The responsivity spread shows a standard deviation of approximately $3 \%$. It is estimated that the pixel outages are in the order of $1-2 \%$ and the pixel are statistically distributed as single pixels without large clusters. Also first dual band LWIR/VLWIR photodiodes [46] and dual band MWIR FPAs [47] have been demonstrated. These very promising results confirm that the antimonide SL technology is now competing with MBE $\mathrm{HgCdTe}$ dual colour technology.

\section{Conclusions}

The future applications of IR detector systems require:

- higher pixel sensitivity,

- further increase in pixel density,

- cost reduction in IR imaging array systems due to less cooling sensor technology combined with integration of detectors and signal processing functions (with much more on-chip signal processing),

- improvement in functionality of IR imaging arrays through development of multispectral sensors.

Array sizes will continue to increase but perhaps at a rate that falls below the Moore's Law curve. An increase in array size is already technically feasible. However, the market forces that have demanded larger arrays are not as strong now that the megapixel barrier has been broken.
For many systems, such as night-vision goggles, the IR image is viewed by the human eye, which can discern resolution improvements only up to about one megapixel, roughly the same resolution as high-definition television. Most high-volume applications can be completely satisfied with a format of $1280 \times 1024$. Although wide-area surveillance and astronomy applications could make use of larger formats, funding limits may prevent the exponential growth that was seen in past decades.

Third generation infrared imagers are beginning a challenging road to development. For multiband sensors, boosting the sensitivity in order maximize identification range is the primary objective. It is predicted that $\mathrm{HgCdTe}$ technology will continue in the future to expand the envelope of its capabilities because of its excellent properties. Despite serious competition from alternative technologies and slower progress than expected, $\mathrm{HgCdTe}$ is unlikely to be seriously challenged for high-performance applications, applications requiring multispectral capability and fast response. However, the nonuniformity is a serious problem in the case of LWIR and VLWIR HgCdTe detectors. For applications that require operation in the LWIR band as well as multi-colour MWIR/LWIR/VLWIR bands most probably $\mathrm{HgCdTe}$ will not be the optimal solution. Type II InAs/ GaInSb superlattice structure is a relatively new alternative IR material system and has great potential for LWIR/ VLWIR spectral ranges with performance comparable to $\mathrm{HgCdTe}$ with the same cutoff wavelength. This material system is also very promising for uncooled LWIR applications.

Based on the breakthrough of Sb-based type II SLS technology it is obvious that this material system is in position to provide high thermal resolution for short integration times comparable to $\mathrm{HgCdTe}$. The fact that Sb-based superlattices are processed close to standard III-V technology rises the potential to be more competitive due to lower costs in series production. The potential low cost compared to $\mathrm{HgCdTe}$ is that it can leverage investments in lasers and transistors in the Sb-based industry, and has potential commercial market applications in the future.

\section{References}

1. P.R. Norton, "Infrared detectors in the next millennium", Proc. SPIE 3698, 652-665 (1999).

2. P. Norton, J. Campbell, S. Horn, and D. Reago, "Thirdgeneration infrared imagers", Proc. SPIE 4130, 226-236 (2000).

3. P.R. Norton, "Third-generation sensors for night vision", Opto-Electron. Rev. 14, 1-10 (2006).

4. A. Rogalski, "Optical detectors for focal plane arrays", Opto-Electron. Rev. 12, 221-245 (2004).

5. P. Norton, "HgCdTe infrared detectors", Opto-Electron. Rev. 10, 159-174 (2002).

6. A. Rogalski, "HgCdTe infrared detector material: History, status, and outlook", Rep. Prog. Phys. 68, 2267-2336 (2005).

7. M.Z. Tidrow, W.A. Beck, W.W. Clark, H.K. Pollehn, J.W. Little, N.K. Dhar, P.R. Leavitt, S.W. Kennerly, D.W. Beekman, A.C. Goldberg, and W.R. Dyer, "Device physics 
and focal plane applications of QWIP and MCT", OptoElectron. Rev. 7, 283-296 (1999).

8. S.D. Gunapala and S.V. Bandara, "GaAs/AlGaAs based quantum well infrared photodetector focal plane arrays", in Handbook of Infrared Detection Technologies, pp. 83-119, edited by M. Henini and M. Razeghi, Elsevier, Oxford, 2002.

9. A. Rogalski, "Quantum well photoconductors in infrared detectors technology", J. Appl. Phys. 93, 4355-4391 (2003).

10. A. Rogalski, "Assessment of $\mathrm{HgCdTe}$ photodiodes and quantum well infrared photoconductors for long wavelength focal plane arrays", Infrared Phys. Technol. 40, 279-294 (1999).

11. L. Bürkle and F. Fuchs, "InAs/(GaIn)Sb superlattices: a promising material system for infrared detection", in Handbook of Infrared Detection and Technologies, pp. 159-189, edited by M. Henini and M. Razeghi, Elsevier, Oxford, 2002.

12. W. Cabanski, K. Eberhardt, W. Rode, J. Wendler, J. Ziegler, J. Fleißner, F. Fuchs, R. Rehm, J. Schmitz, H. Schneider, and M. Walther, " 3 rd gen focal plane array IR detection modules and applications", Proc. SPIE 5406, 184-192 (2005).

13. R. Rehm, M. Walther, J. Schmitz, J. Fleißner, F. Fuchs, J. Ziegler, and W. Cabanski, "InAs/GaSb superlattice focal plane arrays for high-resolution thermal imaging", OptoElectron. Rev. 14, 19-24 (2006).

14. A. Rogalski and P. Martyniuk, "InAs/GaInSb superlattices as a promising material system for third generation infrared detectors", Infrared Phys. Technol. 48, 39-52 (2006).

15. A.W. Hoffman, P.L. Love, and J.P. Rosbeck, "Mega-pixel detector arrays: Visible to 28 um", Proc. SPIE 5167, 194 203 (2004).

16. A. Hoffman, "Semiconductor processing technology improves resolution of infrared arrays", Laser Focus World 42, 81-84 (2006).

17. D. Reago, S. Horn, J. Campbell, and R. Vollmerhausen, "Third generation imaging sensor system concepts", Proc. SPIE, 3701, 108-117 (1999).

18. L.J. Kozlowski and W.F. Kosonocky, "Infrared detector arrays", in Handbook of Optics, Chap. 23, edited by M. Bass, E.W. Van Stryland, D.R. Williams, and W.L. Wolfe, McGraw-Hill, Inc. New York, 1995.

19. S. Horn, P. Norton, K. Carson, R. Eden, and R. Clement, "Vertically-integrated sensor arrays - VISA", Proc. SPIE 5406, 332-340 (2004).

20. R. Balcerak and S. Horn, "Progress in the development of vertically-integrated sensor arrays", Proc. SPIE 5783, 384 391 (2005).

21. E.P.G. Smith, L.T. Pham, G.M. Venzor, E.M. Norton, M.D Newton, P.M. Goetz, V.K. Randall, A.M. Gallagher, G.K. Pierce, E.A. Patten, R.A. Coussa, K. Kosai, W.A. Radford, L.M. Giegerich, J.M. Edwards, S.M. Johnson, S.T. Baur, J.A. Roth, B. Nosho, T.J. De Lyon, J.E. Jensen, and R.E. Longshore, "HgCdTe focal plane arrays for dual-colour mid- and long-wavelength infrared detection", J. Electron. Mater. 33, 509-516 (2004).

22. W.A. Radford, E.A. Patten, D.F. King, G.K. Pierce, J. Vodicka, P. Goetz, G. Venzor, E.P. Smith, R. Graham, S.M. Johnson, J. Roth, B. Nosho, and J. Jensen, "Third generation FPA development status at Raytheon Vision Systems", Proc. SPIE 5783, 331-339 (2005).
23. A.C. Goldberger, S.W. Kennerly, J.W. Little, H.K. Pollehn, T.A. Shafer, C.L. Mears, H.F. Schaake, M. Winn, M. Taylor, and P.N. Uppal, "Comparison of HgCdTe and QWIP dual-band focal plane arrays", Proc. SPIE 4369, 532-546 (2001).

24. H. Schneider, M. Walther, J. Fleissner, R. Rehm, E. Diwo, K. Schwarz, P. Koidl, G. Weimann, J. Ziegler, R. Breiter, and W. Cabanski, "Low-noise QWIPs for FPA sensors with high thermal resolution", Proc. SPIE 4130, 353-362 (2000).

25. H. Schneider, P. Koidl, M. Walther, J. Fleissner, R. Rehm, E. Diwo, K. Schwarz, and G. Weimann, "Ten years of QWIP development at Fraunhofer", Infrared Phys. Technol. 42, 283-289 (2001).

26. M. Jhabvala, K. Choi, A. Goldberg, A. La, and S. Gunapala, "Development of a $1 \mathrm{k} \times 1 \mathrm{k}$ GaAs QWIP far IR imaging array", Proc. SPIE 5167, 175-185 (2004).

27. S.D. Gunapala, S.V. Bandara, J.K. Liu, C.J. Hill, B. Rafol, J.M. Mumolo, J.T. Trinh, M.Z. Tidrow, and P.D. LeVan, "1024×1024 pixel mid-wavelength and long-wavelength infrared QWIP focal plane arrays for imaging applications", Semicond. Sci. Technol. 20, 473-480 (2005).

28. S.D. Gunapala, S.V. Bandara, J.K. Liu, C.J. Hill, B. Rafol, and J.M. Mumolo, " $640 \times 512$ pixel long-wavelength infrared narrowband, multiband, and broadband QWIP focal plane arrays", IEEE Trans. Electron Devices 50, 2353-2360 (2004).

29. G.J. Brown, F. Szmulowicz, K. Mahalingam, S. Houston, Y. Wei, A. Gon, and M. Razeghi, "Recent advances in InAs/GaSb superlattices for very long wavelength infrared detection", Proc. SPIE 4999, 457-466 (2003).

30. D.L. Smith and C. Mailhiot, "Proposal for strained type II superlattice infrared detectors", J. Appl. Phys. 62, 25452548 (1987).

31. C. Mailhiot and D.L. Smith, "Long-wavelength infrared detectors based on strained InAs-GaInSb type-II superlattices", J. Vac. Sci. Technol. A7, 445-449 (1989).

32. G. Bastard, Wave Mechanics Applied to Semiconductor Heterostructures, Monographies de Physiques Series, Halsted Press, New York, 1988.

33. J.P. Omaggio, J.R. Meyer, R.J. Wagner, C.A. Hoffman, M.J. Yang, D.H. Chow, and R.H. Miles, "Determination of band gap and effective masses in InAs/ $\mathrm{Ga}_{1-\mathrm{x}} \mathrm{In}_{\mathrm{X}} \mathrm{Sb}$ superlattices", Appl. Phys. Lett. 61, 207-209 (1992).

34. C.A. Hoffman, J.R. Meyer, E.R. Youngdale, F.J. Bartoli, R.H. Miles, and L.R. Ram-Mohan, "Electron transport in InAs/ $\mathrm{Ga}_{1-\mathrm{x}} \mathrm{In}_{\mathrm{x}} \mathrm{Sb}$ superlattices", Solid State Electron. 37, 1203-1206 (1994).

35. C.H. Grein, P.M. Young, and H. Ehrenreich, "Minority carrier lifetimes in ideal InGaSb/InAs superlattice", Appl. Phys. Lett. 61, 2905-2907 (1992).

36. C.H. Grein, P.M. Young, M.E. Flatté, and H. Ehrenreich, "Long wavelength InAs/InGaSb infrared detectors: Optimization of carrier lifetimes", J. Appl. Phys. 78, 7143-7152 (1995).

37. E.R. Youngdale, J.R. Meyer, C.A. Hoffman, F.J. Bartoli, C.H. Grein, P.M. Young, H. Ehrenreich, R.H. Miles, and D.H. Chow, "Auger lifetime enhancement in InAs- $\mathrm{Ga}_{1-\mathrm{x}} \mathrm{In}_{\mathrm{x}} \mathrm{Sb}$ superlattices", Appl. Phys. Lett. 64, 3160-3162 (1994).

38. O.K. Yang, C. Pfahler, J. Schmitz, W. Pletschen, and F. Fuchs, "Trap centers and minority carrier lifetimes in InAs/ 
GaInSb superlattice long wavelength photodetectors", Proc. SPIE 4999, 448-456 (2003).

39. H. Ehrenreich, C.H. Grein, R.H. Miles and M.E. Flatte, "Reply to "Comment on Temperature limits on infrared detectivities of InAs/ $/ \mathrm{In}_{\mathrm{x}} \mathrm{Ga}_{1-\mathrm{x}} \mathrm{Sb}$ superlattices and bulk $\mathrm{Hg}_{\mathrm{x}} \mathrm{Cd}_{1-\mathrm{x}} \mathrm{Te}$ '", [J. Appl. Phys. 80, 2542 (1996)]", J. Appl. Phys. 80, 2545-2546 (1996).

40. J. Piotrowski and A. Rogalski, "Comment on "Temperature limits on infrared detectivities of $\mathrm{InAs} / \mathrm{In}_{\mathrm{x}} \mathrm{Ga}_{1-\mathrm{x}} \mathrm{Sb}$ superlattices and bulk $\mathrm{Hg}_{\mathrm{x}} \mathrm{Cd}_{1-\mathrm{x}} \mathrm{Te}$," [J. Appl. Phys. 74, 4774 (1993)]", J. Appl. Phys. 80, 2542-2544 (1996).

41. J. Piotrowski and A. Rogalski, "Uncooled long wavelength infrared photon detectors", Infrared Physics \& Technol. 46, 115-131 (2004).

42. J.L. Johnson, "The InAs/GaInSb strained layer superlattice as an infrared detector material: An Overview", Proc. SPIE 3948, 118-132 (2000).
43. G.J. Brown, “Type-II InAs/GaInSb superlattices for infrared detectors: an overview", Proc. SPIE 5783, 65-77 (2005).

44. R. Rehm, M. Walther, J. Schmitz, J. Fleißner, F. Fuchs, W. Cabanski, and J. Ziegler, "InAs/(GaIn)Sb short-period superlattices for focal plane arrays", Proc. SPIE 5783, 123-130 (2005).

45. E.H. Aifer, I. Vurgaftman, C.L. Canedy, J.G. Tischler, J.H. Warner, E.M. Jackson, and J.R. Meyer, "W-Structured type-II superlattices based long-wave infrared photodiodes with high dynamic impedance", to be published.

46. E.H. Aifer, J.G. Tischler, J.H. Warner, I. Vurgaftman, and J.R. Meyer, "Dual band LWIR/VLWIR type-II superlattice photodiodes", Proc. SPIE 5783, 112-122 (2005).

47. R. Rehm, M. Walther, H. Schneider, J. Fleißner, J. Schmitz, J. Ziegler, W. Cabanski, and R. Breiter, "Bispectral thermal imaging with quantum-well infrared photodetectors and InAs/ GaSb type II superlattices", Proc. SPIE 6206, paper 34. 